\title{
UnA REGIONALIZACIÓN DE LA FAJA VolCÁNICA TranSMEXICANA CON BASE EN SU RIQUEZA FLORÍSTICA
}

\author{
Mario E. Suárez-Mota ${ }^{1,3}{ }^{3}$ Oswaldo Téllez-Valdés ${ }^{1}$, Rafael LiRa-Saade ${ }^{1}$ y \\ JOSÉ LUIS VILLASEÑOR \\ 'Unidad de Biotecnología y Prototipos, Facultad de Estudios Superiores Iztacala \\ Universidad Nacional Autónoma de México, Tlalnepantla, Estado de México, México \\ ${ }^{2}$ Instituto de Biología, Universidad Nacional Autónoma de México, México, D.F., México \\ ${ }^{3}$ Autor para la correspondencia: tonatiu969@hotmail.com
}

\begin{abstract}
Resumen: Se propone una regionalización de la provincia morfotectónica de la Faja Volcánica Transmexicana basada en la distribución geográfica de 4,434 especies de plantas vasculares, pertenecientes a 999 géneros y 47 familias. Mediante la clasificación de una matriz de datos estructurada, con la distribución de los registros de las especies sobre una rejilla de celdas de 30' de latitud y longitud (aproximadamente 2,500 km²) se calcularon las similitudes florísticas entre las celdas utilizando el coeficiente de similitud de Sorensen-Dice. A partir de los agrupamientos de celdas mediante el método de enlace completo se definieron cuatro unidades florísticas (grupos de celdas) dentro de la Faja Volcánica Transmexicana. Un par de estas unidades no son continuas, pues muestran disyunciones entre las otras unidades, debido a accidentes orográficos que las separan. Igualmente, las unidades difieren en tamaño y composición florística y ocupan espacios geográficos particulares de la Faja Volcánica Transmexicana, en función de los parámetros abióticos que las caracterizan.
\end{abstract}

Palabras clave: distribución geográfica, elementos florísticos, unidades florísticas.

Abstract: We propose the regionalization of the Transmexican Volcanic Belt morphotectonic province, based on the distribution of 4,434 of vascular plant species belonging to 999 genera and 47 families. Regionalization was done using a data matrix with species recorded in grid-cells of 30' latidude and longitude (about 2,500 km²) all along the Transmexican Volcanic Belt morphotectonic province. Based on clusters obtained with the complete linkage method, cells in the Transmexican Volcanic Belt were grouped in four floristic units; a couple of them show disjunct distribution due mostly to the orographic accidents that separate them. Likewise, the units differ in size and floristic composition and they occupy particular geographic positions inside the Transmexican Volcanic Belt, as response to the abiotic parameters that characterize them.

Key words: floristic elements, floristic units, geographical distribution.

L a distribución de la biodiversidad en el planeta es discontinua. Algunos países donde se concentran importantes cantidades de especies se han identificado como megadiversos (Mittermeier, 1988; Akeroyd y Synge, 1992) y México está considerado entre estos países por su alta diversidad biológica (Rzedowski, 1978; Mittermeier, 1988; Villaseñor, 2003). El territorio mexicano ha funcionado como centro de evolución de linajes, debido a su gran diversidad climática, fisiográfica, geológica y edáfica. Esto se ve reflejado en condiciones que permiten observar una alta diversidad alfa, que a su vez, favorece la notable diversidad beta (Rzedowski, 1991) y gama encontradas en el país.
En México se encuentra una diversidad vegetal excepcional, en la que resalta la combinación de elementos boreales y meridionales que han propiciado el origen de la riqueza florística que se distribuye en el país (Rzedowski, 1991). Los principales factores que favorecen la riqueza florística de México son la variedad de climas y las condiciones fisiográficas. Además, su situación geográfica entre las dos regiones continentales boreal y meridional ha sido escenario de migraciones de plantas que constituyen una zona de influencia mixta de los elementos florísticos neotropical y holártico (Rzedowski, 1978). Existen clasificaciones biogeográficas que han dividido esta diversidad vegetal en 
diferentes regiones o provincias; por ejemplo, Rzedowski (1978) divide al país en 17 provincias florísticas agrupadas en cuatro regiones, una de las cuales (Región Mesoamericana de Montaña) incluye a la Faja Volcánica Transmexicana (en adelante FVT), zona que resalta por una flora constituida por alrededor de 5,139 especies distribuidas principalmente en los bosques templados que lo constituyen (Villaseñor y Ortiz, 2007).

Cualquier flora es la representación de los grupos de plantas que se encuentran en un área determinada (McLaughlin, 1994). Esta área puede ser referida a distintas escalas, como local, regional o nacional. El uso de clasificaciones realizadas a partir de agrupamientos formados por la distribución de la diversidad florística ha permitido diferenciar las diversas floras que se encuentran en México y a su vez agruparlas en provincias florísticas (Rzedowski y ReynaTrujillo, 1990).

Algunos autores, como Rzedowski (1973, 1978), Morafka et al. (1992), Arriaga et al. (1997), Contreras-Medina y Eliosa-León (2001) o Morrone (2001, 2005, 2006), han propuesto esquemas de regionalización del país en diferentes provincias y subprovincias. Entre ellas, resalta la FVT como una provincia, delimitada con base en su composición florística y faunística analizada desde distintas perspectivas metodológicas como los análisis de parsimonia de endemismos, la cladística, la fenética y la panbiogeografía.

El conocimiento sobre los patrones de distribución geográfica de las especies se acumula continuamente. Esto permite rehacer análisis de los límites geográficos y sus relaciones bióticas, que permiten proponer nuevas propuestas de regionalización. La definición y delimitación de las áreas geográficas donde se distribuyen las especies siempre será motivo de discusión, debido a que existen diferentes criterios en el conocimiento biológico y ambiental, que están sujetos a continuos cambios (Ochoa-Tejeda, 2001).

Una regionalización es el proceso de agrupar u ordenar la información de un número determinado de unidades territoriales, con el propósito de evaluar sus similitudes y relaciones. Existen diferentes formas de regionalizar biogeográficamente un territorio; por ejemplo, Morrone y Márquez (2003) y Morrone $(2005,2006)$ proponen un esquema de regionalización de México compuesto por 14 provincias, agrupadas en dos regiones y una zona de transición. La región Neártica incluye cinco provincias y la Neotropical cuatro provincias, mientras que la zona de transición la definen como el "Componente Mexicano de Montaña", caracterizado por otras cinco provincias: (1) Sierra Madre Occidental, (2) Sierra Madre Oriental, (3) Faja Volcánica Transmexicana, (4) Depresión del Balsas y (5) Sierra Madre del Sur.

El Componente Mexicano de Montaña es equivalente a la Zona de Transición Mexicana (Halffter, 1976, 2003; Morrone, 2005, 2006), área que exhibe una alta diversidad biológica como resultado de importantes procesos de especiación ocurridos por la notable actividad volcánica en dicha zona. Adicionalmente, en ella entran en contacto las regiones Neártica y Neotropical. Todo ello, junto con su variabilidad climática, edáfica o geológica, favorece condiciones responsables de la gran riqueza de especies observada en esta zona. La FVT es considerada como la zona de transición entre estas dos regiones y además, conecta transversalmente a la Sierra Madre Oriental con la Sierra Madre Occidental (Vivó, 1943; Darlington, 1957).

La región de la FVT, ha sido estrechamente relacionada por su semejanza florística y faunística con otras regiones, como la Sierra Madre Oriental, la Sierra Madre del Sur y el Valle de Tehuacán-Cuicatlán (Delgadillo et al., 2003; Villaseñor, 2004; Escalante et al., 2005; Morrone, 2005; Suárez-Mota, 2006). Sin embargo, existen pocos trabajos que consideren los patrones de distribución geográfica de la flora y fauna que permitan proponer divisiones biogeográficas dentro de la FVT para complementar o enriquecer aquellas que previamente se han definido. Por ejemplo, una propuesta es la de las provincias avifaunísticas que hacen Navarro-Sigüenza et al. (2007) y otra de Escalante et al. (2007), que dividen la FVT en dos distritos, uno oriental y otro occidental; ambas propuestas se definieron a partir de análisis de la distribución potencial de las especies. Sánchez-Cordero et al. (2005), a partir de la distribución geográfica de mamíferos, dividen a la FVT en tres regiones, una oriental, otra central y una occidental. Mediante un análisis panbiogeográfico, utilizando 156 especies pertenecientes a cinco grupos diferentes (aves, mamíferos, reptiles, helechos y encinos), Torres y Luna (2007) proponen cinco distritos en esta provincia.

La FVT presenta una riqueza excepcional de algunos grupos de plantas. Por ejemplo, está documentado que existen más especies del género Sedum en dicha provincia que en cualquier otra parte de América del Norte (Clausen, 1959). Otro ejemplo es el género Quercus, del que se han reportado 75 de sus especies dentro de los estados que conforman la FVT, cifra que corresponde a $46 \%$ del total de las especies reportadas en México (Valencia-A., 2004). Un tercer ejemplo es el género Pinus, que forma parte de los géneros que tienen en México uno de sus principales centros de diversificación (Rzedowski y Calderón de Rzedowski, 1993); en la FVT se tienen registradas 21 (50\%) de las 42 especies de este género que se distribuyen en México (Farjon y Styles, 1997). La FVT no solamente destaca por su alto contenido de especies de plantas, sino que también presenta un alto número de especies endémicas (Fa y Morales, 1991, Villaseñor et al., 2007). Por otra parte, esta provincia es la región del país que mayor atención ha recibido; en su territorio se han realizado diversos estudios, sobre todo aquellos relacionados con su geología y geofísica por cuestiones de seguridad, debido a que es una región geológica o vulcanológicamente activa y donde se concentra la mayor parte de la población e infraestructura del país (Gómez-Tuena et al., 2005).

Para la FVT se tienen distintas propuestas de regiona- 
lización, entre las que desatacan las de Alcorta (1964) y Cuanalo et al. (1989), quienes lo definen como una "región natural", sin explicar los criterios específicos utilizados para sustentarlo. Otros autores la definen como una provincia fisiográfica, caracterizada por sus rasgos geomorfológicos y por sus formas de relieve (Raisz, 1964; Lugo-Hubp, 1990). Otros más como una provincia morfotectónica (Mooser, 1972; López-Ramos, 1979; Morán-Zenteno, 1984; OrtegaGutiérrez et al., 1992; Ferrari et al., 1999; Ferrari, 2000). $\mathrm{Si}$ a estas contribuciones geográfico-geológicas, se suman los esquemas de regionalización biogeográfica, ecológica, faunística y florística, como los estudios de Rzedowski (1978), Rzedowski y Reyna-Trujillo (1990), Casas-Andreu y Reyna-Trujillo (1990), Goldman y Moore (1945), Ramírez-Pulido y Castro-Campillo (1990), Ferrusquía-Villafranca (1990) y Smith (1941), se comprende la dificultad de tener una delimitación concluyente del área que ocupa la FVT. Sin embargo, todas estas propuestas pueden contrastarse para hacer un consenso que permita encontrar sus coincidencias y discrepancias.

En México existen 2,804 géneros de plantas vasculares (Villaseñor, 2004), de los cuales 1,348 (48.1\%) se registran en la FVT (Villaseñor, 2004). Estos géneros se distribuyen en diferentes tipos de vegetación que se encuentran en la FVT; entre los principales se encuentran los bosques de pino, de encino, de oyamel, los tropicales caducifolios, el húmedo de montaña y el pastizal (Rzedowski, 1978).

Este trabajo tiene como objetivo proponer una regionalización florística de la FVT, a partir de la distribución de su flora y mediante un análisis multivariado. Con ello se espera reconocer áreas con importantes niveles de similitud florística, que permitan ser identificadas como regiones florísticas (Birks, 1976).

\section{Método}

Área de estudio. Se utilizó el polígono correspondiente a la provincia de la Faja Volcánica Transmexicana delimitado en la regionalización geomorfológica de México (FerrusquíaVillafranca, 1990). Esta provincia está incluida en el territorio de 16 estados del país (CONABIO, 1997). Se extiende desde las costas del Pacífico, en San Blas, Nayarit y Bahía de Banderas, Jalisco, hasta las costas del Golfo de México en Palma Sola, Veracruz, por los $19^{\circ}$ de latitud N (Demant, 1978). La provincia tiene aproximadamente $1,000 \mathrm{~km}$ de longitud y una amplitud irregular, que oscila entre los $80 \mathrm{y}$ $230 \mathrm{~km}$, cuyas coordenadas extremas son: al norte $21^{\circ} 38^{\prime}$ $24^{\prime \prime}$, al sur $18^{\circ} 23^{\prime} 24^{\prime \prime}$, al este $96^{\circ} 22^{\prime} 12^{\prime \prime}$ y al oeste $105^{\circ}$ 45' 00" (Gómez-Tuena et al., 2005; Figura 1).

Para la regionalización se recopilaron datos de especies de la flora presente en la FVT. Los criterios de selección de las especies fueron: (1) pertenecer a familias de plantas que

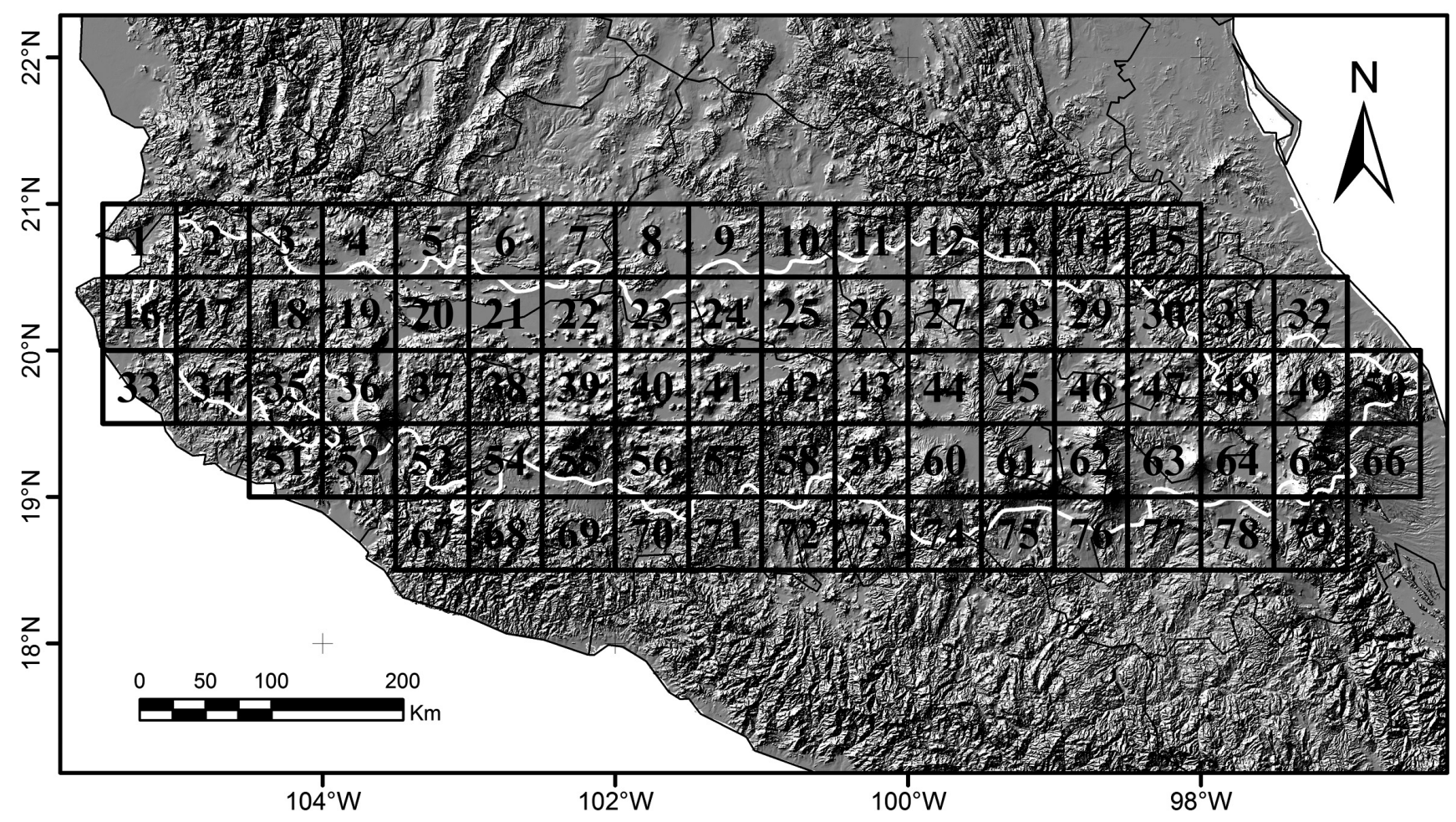

Figura 1. Área de estudio (polígono de la Faja Volcánica Transmexicana, CONABIO, 1997) dividido en 79 Unidades Geográficas Operativas, cada una de ellas de 30' de latitud y longitud. 
se distribuyen en los tipos de vegetación que se registran en la FVT, (2) estar registradas en colecciones biológicas y/o bases de datos disponibles y (3) que sus localidades de colecta estén georreferenciadas. Con estos criterios fueron seleccionadas especies documentadas en las bases de datos incluidas en la Red Mundial de Información sobre Biodiversidad (REMIB), disponible en la página web de la Comisión Nacional para el Conocimiento y Uso de la Biodiversidad (Conabio). Con los límites del polígono de la provincia morfotectónica de la FVT y con los datos de las especies (nombre científico y georreferencias), se estructuró una base de datos, la cual se organizó utilizando el programa Microsoft Access 2007.

Los datos obtenidos de la REMIB fueron revisados previamente a su uso. De esta manera, los nombres de las especies se compararon con la información contenida en catálogos y fichas taxonómicas que se han producido en diversos herbarios, tanto nacionales como del extranjero y publicadas en páginas electrónicas; por ejemplo, Trópicos (http://www. tropicos.org), IPNI (http://www.ipni.org/index.html) o ITIS (http://www.itis.gov/servlet/). También se revisaron datos incluidos en otras fuentes, como en el catálogo florístico del bosque húmedo de montaña en México (Villaseñor, 2010). De esta manera se redujo notablemente la sinonimia y se corrigió la ortografía de los nombres. Por otra parte, se hizo una evaluación geográfica, que consistió en el mapeo de los registros utilizando el sistema de información geográfica (SIG) ArcView 3.2 (ESRI, 2000); con ello se corroboró que los registros coincidieran con los estados y localidades donde han sido registrados y con ello verificar que los patrones de distribución de las especies estuvieran dentro del polígono de la FVT.

La descripción biogeográfica de la flora se hizo con base en dos términos de análisis de los datos, uno denominado Regiones Florísticas, empleado para determinar áreas de composición florística similar; y otro, Elementos Florísticos, que determina grupos de especies con distribución geográfica similar (Birks, 1976). Los análisis multivariados ayudaron a determinar tales Regiones Florísticas y reconocer los Elementos Florísticos que las componen (Birks, 1976). Para realizar los análisis, la FVT se dividió en unidades más pequeñas, conocidas como Unidades Geográficas Operativas (UGOs).

La Unión Mundial para la Naturaleza (UICN) considera que una buena estrategia de análisis de los patrones de distribución de las especies es mediante el uso de cuadros o celdas en que se divide la región de estudio (UICN, 2001). El tamaño de la cuadrícula en que se dividió la FVT fue determinado siguiendo las recomendaciones de la misma UICN. En este trabajo se calculó la distancia de los sitios extremos conocidos para cada especie; tomando el $10 \%$ de la distancia extrema de cada especie y promediando todos estos valores se obtuvo un valor que fue utilizado para determinar el tamaño de celda (Suárez-Mota y Villaseñor,
2011; Suárez-Mota, 2012). De esta manera, la FVT fue dividida en celdas de 30' de latitud y longitud, pues la distancia promedio de todas las especies analizadas fue de $489.9 \mathrm{~km}$ y el $10 \%$ de este valor corresponde aproximadamente con una celda de $50 \mathrm{~km}$ de latitud y longitud; este valor es muy cercano a una celda de 30' de latitud y longitud (ca. 48 km por lado). Utilizando este tamaño de cuadro, la FVT fue dividida en 79 UGOs (Figura 1).

Las UGOs que incluyeron menos del 50\% de la superficie de un cuadro completo, fueron unidas con celdas vecinas. Esta fusión se hizo considerando principalmente la distribución de las especies al analizarlas en el SIG. Dicha fusión de celdas resultó en un rearreglo que dio como resultado 48 UGOs (Figura 2), pues muchas celdas de los extremos norte y sur fueron colapsadas con sus vecinas siguiendo una orientación latitudinal. Con ello se trató de disminuir la fuerte discrepancia en los valores de riqueza propiciada por la desigual cantidad de registros de especies en cada una de las UGOs. Con estas 48 unidades y utilizando el SIG se calculó la riqueza de especies en cada una; los valores obtenidos se ordenaron en cuartiles, y aquellas UGOs cuyo valor de riqueza fue menor al del primer cuartil se eliminaron del análisis. También se discriminaron las especies registradas en una sola UGO.

Una vez definido el tamaño de la retícula y la composición de especies, se estructuró una matriz de presencia-ausencia (datos binarios) con las especies registradas en cada celda. Para ello, se intersectaron en el SIG los registros de distribución de las especies con el polígono de la FVT dividido en celdas. En la matriz, las especies se ordenaron como filas y las UGOs como columnas.

El análisis multivariado se hizo empleando el coeficiente de similitud de Sorensen-Dice implementado en el software NTSyS 2.0 (Rholf, 2002). El agrupamiento de las UGOs por sus similitudes florísticas se realizó utilizando el método de agrupamiento enlace completo. Con esta clasificación se determinaron las Unidades Florísticas. Para identificar los Elementos Florísticos se utilizó el mapa de las Unidades Florísticas (Grupos de UGOs formados en el primer análisis), con el cual se intersectaron los registros de las especies utilizando nuevamente el SIG.

Para evaluar la distribución de las especies se hizo un análisis de su distribución en los tipos de vegetación que se encuentran en la FVT. Estos tipos de vegetación se delimitaron geográficamente con el mapa de la serie III de INEGI (INEGI, 2005) y su coincidencia en 50 diferentes zonas climáticas definidas como dominios climáticos, que permiten reconocer la variabilidad climática de la FVT (Suárez-Mota, 2012).

\section{Resultados}

La Faja Volcánica Transmexicana es una de las regiones con mayor diversidad florística en México. Se han reportado al 


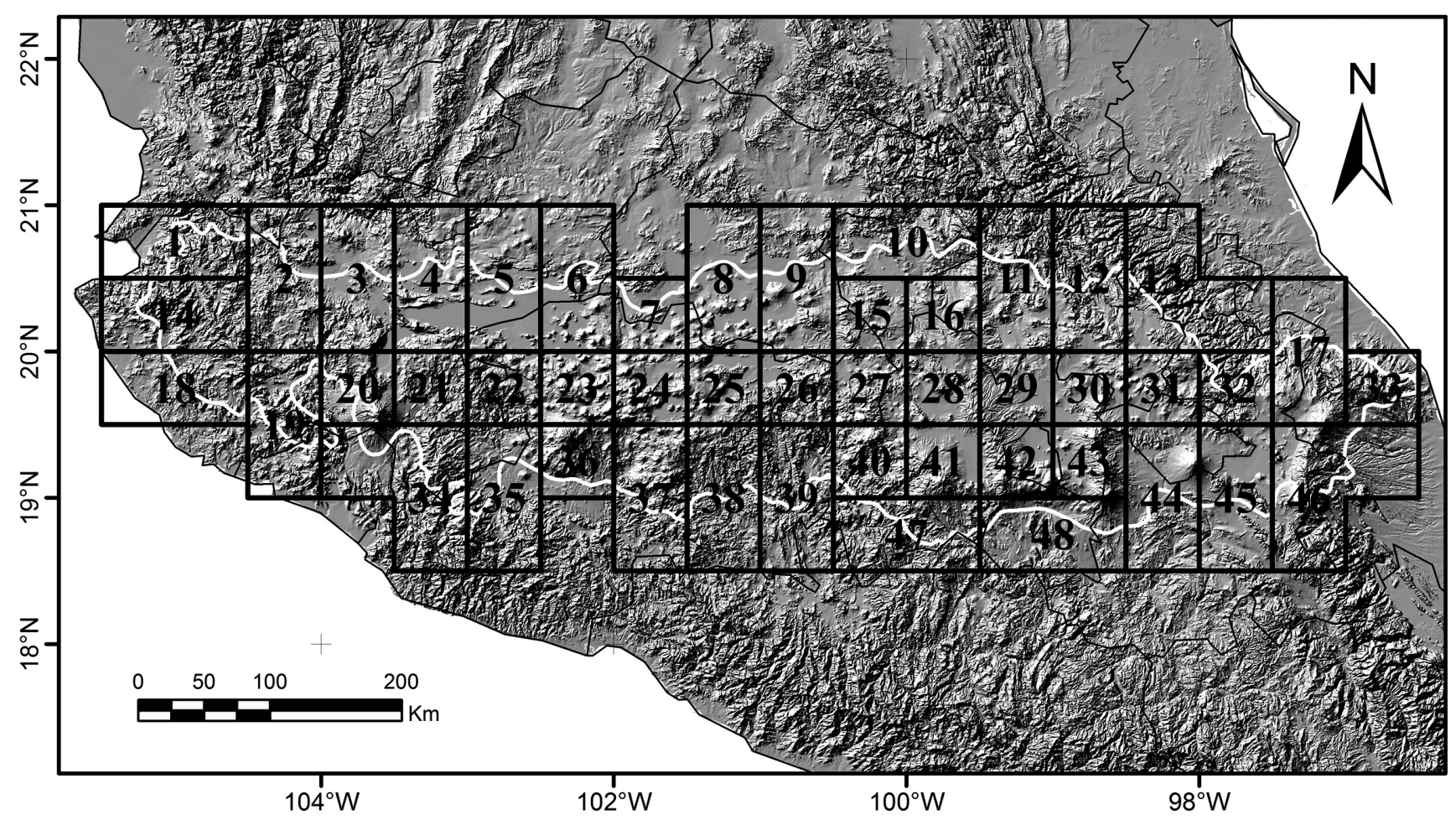

Figura 2. Mapa de la FVT modificado al unir UGOs con superficie menor del $50 \%$ con su cuadro vecino.

menos 5,139 especies vasculares en los bosques templados de la provincia (Villaseñor y Ortiz, 2007). En este trabajo se analizaron 4,055 especies, agrupadas en 999 géneros y 47 familias (Cuadro 1); tales cifras representan $78.9 \%$ de las estimadas por Villaseñor y Ortiz (2007). Las familias con mayor número de especies registradas son, en primer lugar Asteraceae (761, 18.8\%), en segundo lugar Fabaceae (592, $14.6 \%$ ) y en tercer lugar Poaceae $(588,14.5 \%)$. Las familias con menos especies registradas en la FVT son Hamamelidaceae, Magnoliaceae y Podocarpaceae con 4, 6 y 3 especies respectivamente (Cuadro 1).

La distribución de las especies en las UGOs en que se dividió la FVT (Figura 2) es bastante heterogénea. La UGO con mayor número de especies fue la 33 (1,601 especies, 39.5\% de la riqueza total), mientras que en la UGO 39 sólo se registraron 62 especies (1.5\%); en promedio se registran 267 especies por UGO (desviación estándar $=31.8$; Cuadro 2).

La mayoría de los taxones de la FVT muestran una distribución restringida; las frecuencias nos señalan que el primer cuartil comprende las especies que se distribuyen en una y dos celdas. Por lo tanto, 1,633 especies caen en este grupo, por lo que podríamos decir que este conjunto de especies constituye la rareza de la FVT $(40.3 \%)$, con una mediana de tres. Con el arreglo de las frecuencias en clases mediante la regla de Sturges (Daniel, 1982) se encontró que $79.3 \%$ de las especies se distribuyen en siete UGOs o menos. De ellas, $789(24.5 \%)$ solamente se han registrado en una sola de las 48 UGOs. Por otra parte, $20.3 \%$ del total de las 4,055 especies analizadas se distribuyen entre ocho y 30 UGOs y únicamente $0.3 \%$ se distribuyen en 30 UGOs o más (Figura 3).

La superficie total de la provincia morfotectónica de la FVT es de $163,015 \mathrm{~km}^{2}$; de ellos, la cobertura vegetal es de $58,274.2 \mathrm{~km}^{2}(35.7 \%)$, mientras que el resto $104,740.8$ $\mathrm{km}^{2}(64.2 \%)$ ha sido ocupado para establecer zonas urbanas, agrícolas, ganaderas o industriales, lo que ha influido notablemente en la reducción del área de distribución de las especies, no sólo de esta región sino de todo el país (Cuadro 3 ). En promedio se registraron diez tipos de vegetación por UGO, observándose una correlación relativamente baja $(r=$ $0.45 ; P<0.005)$ entre la riqueza de especies y los tipos de vegetación. En el cuadro 3 se indica la riqueza de especies reconocida en los tipos de vegetación utilizados para el análisis. Más de la mitad de las especies se registraron en más de un tipo de vegetación; domina, por el número de especies, el pastizal inducido $(1,460)$, seguido por el bosque de pino-encino (1,369 especies), mientras que para el bosque húmedo de montaña se reconocen 977 (Cuadro 3).

En la FVT se observa que en distancias muy cortas ocurren cambios climáticos drásticos, debido a los gradientes altitudinales, a la complejidad topográfica y a su orografía en general, así como a la elevación de las serranías (Greenland, 2005). Tal variabilidad climática se ve reflejada en el número de dominios climáticos encontrados en cada UGO; solamente en dos se registran al menos cinco dominios, mientras que una sola UGO (17) registra 21 diferentes do- 
Mario E. SuÁrez-Mota ET AL.

Cuadro 1. Familias botánicas consideradas para la regionalización florística de la Faja Volcánica Transmexicana. Se indica el número de géneros, especies y registros incluidos.

\begin{tabular}{|c|c|c|c|c|}
\hline No. & Familia & Géneros & Especies & Registros \\
\hline 1 & Acanthaceae & 26 & 84 & 697 \\
\hline 2 & Agavaceae & 9 & 63 & 442 \\
\hline 3 & Apiaceae & 23 & 53 & 390 \\
\hline 4 & Aquifoliaceae & 1 & 9 & 124 \\
\hline 5 & Araceae & 10 & 20 & 132 \\
\hline 6 & Araliaceae & 6 & 18 & 372 \\
\hline 7 & Asparagaceae & 1 & 7 & 166 \\
\hline 8 & Asteraceae & 166 & 761 & 10,740 \\
\hline 9 & Begoniaceae & 1 & 22 & 422 \\
\hline 10 & Bignoniaceae & 18 & 29 & 183 \\
\hline 11 & Brassicaceae & 20 & 34 & 339 \\
\hline 12 & Bromeliaceae & 7 & 59 & 616 \\
\hline 13 & Burseraceae & 2 & 31 & 488 \\
\hline 14 & Cactaceae & 28 & 99 & 738 \\
\hline 15 & Caprifoliaceae & 5 & 22 & 475 \\
\hline 16 & Caryophyllaceae & 17 & 52 & 1,114 \\
\hline 17 & Celastraceae & 9 & 17 & 180 \\
\hline 18 & Convolvulaceae & 13 & 110 & 1,408 \\
\hline 19 & Crassulaceae & 6 & 54 & 701 \\
\hline 20 & Cucurbitaceae & 26 & 77 & 1,925 \\
\hline 21 & Ericaceae & 18 & 74 & 1,511 \\
\hline 22 & Euphorbiaceae & 23 & 126 & 1,441 \\
\hline 23 & Fabaceae & 93 & 592 & 5,417 \\
\hline 24 & Fagaceae & 3 & 60 & 1,922 \\
\hline 25 & Gesneriaceae & 11 & 24 & 273 \\
\hline 26 & Hamamelidaceae & 3 & 4 & 96 \\
\hline 27 & Lauraceae & 8 & 40 & 395 \\
\hline 28 & Liliaceae & 14 & 21 & 421 \\
\hline 29 & Magnoliaceae & 2 & 6 & 79 \\
\hline 30 & Malvaceae & 30 & 104 & 1,619 \\
\hline 31 & Melastomataceae & 12 & 65 & 891 \\
\hline 32 & Myrsinaceae & 9 & 27 & 289 \\
\hline 33 & Myrtaceae & 10 & 37 & 314 \\
\hline 34 & Orchidaceae & 85 & 321 & 2,541 \\
\hline 35 & Pinaceae & 3 & 30 & 500 \\
\hline 36 & Piperaceae & 3 & 59 & 891 \\
\hline 37 & Plantaginaceae & 1 & 13 & 277 \\
\hline 38 & Poaceae & 126 & 588 & 10,452 \\
\hline 39 & Podocarpaceae & 1 & 3 & 67 \\
\hline 40 & Polypodiaceae & 7 & 67 & 1,048 \\
\hline 41 & Pteridaceae & 18 & 85 & 1,258 \\
\hline 42 & Rubiaceae & 49 & 191 & 2,875 \\
\hline 43 & Salicaceae & 2 & 15 & 221 \\
\hline 44 & Scrophulariaceae & 39 & 116 & 3,032 \\
\hline 45 & Solanaceae & 20 & 99 & 1,854 \\
\hline 46 & Tiliaceae & 5 & 14 & 105 \\
\hline 47 & Urticaceae & 10 & 32 & 382 \\
\hline
\end{tabular}

Cuadro 2. Total de especies (TS), número de tipos de vegetación (TV) y de dominios climáticos (DM) registradas en cada una de las UGOs en que se dividió la FVT.

\begin{tabular}{|c|c|c|c|}
\hline UGOs & TS & TV & DM \\
\hline 1 & 88 & 10 & 7 \\
\hline 2 & 171 & 7 & 8 \\
\hline 3 & 305 & 11 & 5 \\
\hline 4 & 497 & 11 & 7 \\
\hline 5 & 96 & 6 & 4 \\
\hline 6 & 124 & 6 & 6 \\
\hline 7 & 224 & 9 & 5 \\
\hline 8 & 229 & 7 & 5 \\
\hline 9 & 396 & 7 & 8 \\
\hline 10 & 174 & 9 & 5 \\
\hline 11 & 246 & 10 & 8 \\
\hline 12 & 625 & 13 & 10 \\
\hline 13 & 254 & 10 & 7 \\
\hline 14 & 339 & 10 & 7 \\
\hline 15 & 377 & 7 & 10 \\
\hline 16 & 228 & 10 & 9 \\
\hline 17 & 1343 & 12 & 21 \\
\hline 18 & 65 & 7 & 4 \\
\hline 19 & 491 & 9 & 8 \\
\hline 20 & 320 & 14 & 8 \\
\hline 21 & 338 & 8 & 9 \\
\hline 22 & 163 & 8 & 12 \\
\hline 23 & 302 & 8 & 9 \\
\hline 24 & 854 & 10 & 6 \\
\hline 25 & 904 & 9 & 8 \\
\hline 26 & 735 & 12 & 12 \\
\hline 27 & 554 & 11 & 12 \\
\hline 28 & 140 & 7 & 6 \\
\hline 29 & 731 & 12 & 11 \\
\hline 30 & 731 & 14 & 6 \\
\hline 31 & 171 & 10 & 7 \\
\hline 32 & 168 & 9 & 14 \\
\hline 33 & 1601 & 9 & 10 \\
\hline 34 & 480 & 12 & 7 \\
\hline 35 & 63 & 8 & 5 \\
\hline 36 & 382 & 9 & 9 \\
\hline 37 & 645 & 10 & 11 \\
\hline 38 & 235 & 8 & 15 \\
\hline 39 & 62 & 6 & 11 \\
\hline 40 & 459 & 9 & 13 \\
\hline 41 & 289 & 11 & 11 \\
\hline 42 & 867 & 14 & 12 \\
\hline 43 & 759 & 12 & 13 \\
\hline 44 & 389 & 12 & 13 \\
\hline 45 & 149 & 12 & 10 \\
\hline 46 & 1009 & 13 & 12 \\
\hline 47 & 275 & 10 & 8 \\
\hline 48 & 489 & 12 & 12 \\
\hline
\end{tabular}


Cuadro 3. Tipos de vegetación reportados en la FVT (INEGI, 2007). Se indica el área y porcentaje que ocupa cada uno de ellos, así como el número de especies y de registros.

\begin{tabular}{|c|c|c|c|c|c|}
\hline Clave & Tipo de Vegetación & Área & $\%$ de la FVT & $\begin{array}{l}\text { Total de } \\
\text { especies }\end{array}$ & $\begin{array}{l}\text { Total de } \\
\text { registros }\end{array}$ \\
\hline BE & Bosque de Encino & $9,687.7$ & 5.94 & 1,387 & 4,418 \\
\hline BEP & Bosque de Encino-Pino & $2,827.4$ & 1.73 & 746 & 1,649 \\
\hline $\mathrm{BO}$ & Bosque de Oyamel & $1,143.8$ & 0.70 & 387 & 893 \\
\hline $\mathrm{BP}$ & Bosque de Pino & $5,487.9$ & 3.37 & 1,277 & 5,384 \\
\hline BPE & Bosque de Pino-Encino & $11,617.9$ & 7.13 & 1,369 & 4,498 \\
\hline BT & Bosque de Tascate & 489.3 & 0.30 & 11 & 11 \\
\hline BMM & Bosque Húmedo de Montana & $1,401.2$ & 0.86 & 977 & 2,309 \\
\hline MCR & Matorral Crasicaule & $1,987.8$ & 1.22 & 355 & 631 \\
\hline MDR & Matorral Desértico Rosetofilo & 658 & 0.40 & 414 & 975 \\
\hline MSUM & Matorral Submontano & 76.5 & 0.05 & 12 & 12 \\
\hline $\mathrm{PH}$ & Pastizal Halófilo & 334.8 & 0.21 & 34 & 39 \\
\hline $\mathrm{PI}$ & Pastizal Inducido & $10,156.8$ & 6.23 & 1,460 & 4,077 \\
\hline PAMO & Pradera de Alta Montana & 139.6 & 0.09 & 141 & 242 \\
\hline SAP & Selva Alta Perennifolia & 75.9 & 0.05 & 10 & 13 \\
\hline SBC & Selva Baja Caducifolia & $11,298.6$ & 6.93 & 918 & 1,907 \\
\hline SMSUC & Selva Mediana Subcaducifolia & 539.4 & 0.33 & 12 & 13 \\
\hline TUL & Tular & 167.8 & 0.10 & 2 & 7 \\
\hline \multirow[t]{2}{*}{$\mathrm{VH}$} & Vegetación Halófila & 183.8 & 0.11 & 33 & 38 \\
\hline & TOTAL & $58,274.2$ & 35.75 & -- & 27,116 \\
\hline
\end{tabular}

minios (Cuadro 2). Se encontró una correlación positiva entre el número de dominios climáticos y tipos de vegetación por UGO $(r=67.8, P<0.005)$; en promedio se registraron 6.7 dominios climáticos por UGO.

La similitud florística entre las UGOs en que se dividió la FVT permite identificar tres grupos principales (Figura 4), definidos como unidades florísticas (Birks, 1976), aunque tales grupos se forman a niveles de similitud por debajo del $10 \%$. El primer grupo está formado por cuatro UGOs $(1,18$, 35 y 39), el segundo grupo incluye 17 UGOs, mientras que al tercer grupo lo conforman 27 UGOs (Figura 4). Diez UGOs del grupo tres $(24,25$ y 26; 29 y $30 ; 42$ y $43 ; 17,46$ y 33) se asociaron por encima del $50 \%$ de similitud y ningún otro conjunto de UGOs alcanza estos valores de similitud. Las UGOs 29 y 30 son las que registran la mayor similitud florís- tica (65.4\%). El segundo grupo se dividió en dos subgrupos: uno (2a) formado por nueve UGOs y otro subgrupo (2b) con ocho UGOs (Figura 4). El tercer grupo también se dividió en dos subgrupos: uno (3a) incluye 23 UGOs, mientras que el otro (3b) lo forman cuatro de las UGOs (Figura 4). Estos subgrupos (2a, 2b, 3a y $3 \mathrm{~b}$ ) corresponden con las Unidades florísticas (II y III) respectivamente (Cuadro 4).

Debido a que las UGOs del primer grupo no forman una Unidad geográfica común, sino que están ubicados en zonas disyuntas, entremezclados con UGOs de otros grupos, se reasignaron a los otros dos grupos; de esta manera tres de ellas (1, 18 y 35$)$ se unieron en el subgrupo 2a (Figura 4) y una más (UGO 39) en el subgrupo 3a (Figura 5). Con la segregación de este grupo finalmente se definieron cuatro unidades florísticas: la Unidad I está formada por 12 UGOs

Cuadro 4. Unidades florísticas definidas en la Faja Volcánica Transmexicana y elementos florísticos que las caracterizan. Se indica el número de especies exclusivas a cada unidad, el número de UGOs que las constituyen y el número de tipos de vegetación que se registran en ellas.

\begin{tabular}{ccccc}
\hline $\begin{array}{c}\text { Unidad } \\
\text { florística }\end{array}$ & $\begin{array}{c}\text { (total de } \\
\text { especies) }\end{array}$ & $\begin{array}{c}\text { Elemento florístico } \\
\text { (Especies exclusivas) }\end{array}$ & UGOs & $\begin{array}{c}\text { Tipos de } \\
\text { vegetación }\end{array}$ \\
\hline I & 1,876 & 332 & $1,2,3,4,14,18,19,20,34,35,40,47$ & 14 \\
II & 886 & 31 & $5,6,7,8,10,11,16,22$ \\
III & 2,642 & 586 & $9,12,13,15,21,23,24,25,26,27,28,29$, \\
IV & 2,282 & 764 & $30,31,36,37,38,39,41,42,43,44,45,48$ & 16 \\
\hline
\end{tabular}




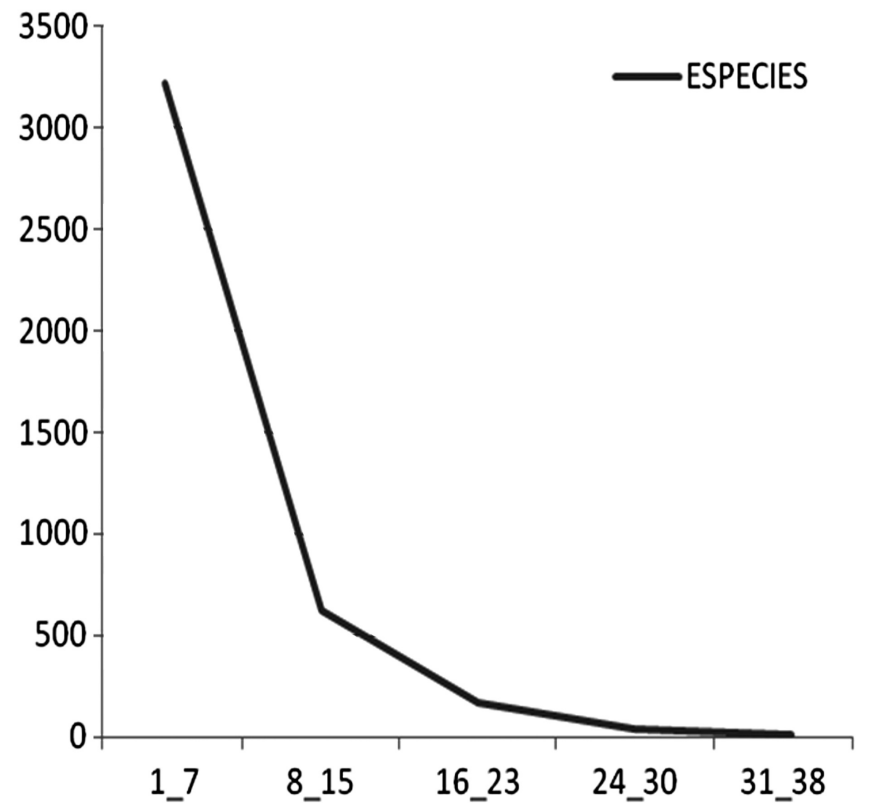

Figura 3. Distribución de las especies analizadas en las 48 UGOs en que se dividió la FVT, agrupadas en clases.

distribuidas en la parte occidental de la FVT. La Unidad II incluye ocho UGOs localizadas en la parte norte, donde la FVT entra en contacto con la Altiplanicie Mexicana (Cuadro 4). La Unidad III agrupa veinticuatro UGOs distribuidas en las partes central, NE y SE de la FVT. Por último, la Unidad IV sólo incluye cuatro UGOs localizadas en el extremo oriental de la FVT (Figura 5).

Aunque cada unidad florística contiene un número parecido de comunidades vegetales, es notoria la diferencia en el número de especies que incluyen (Cuadro 4). Aunque la Unidad I, registra 14 comunidades vegetales, el número de especies $(1,876)$ que registra es menor si se compara, por ejemplo, con la riqueza observada en la Unidad IV $(2,282)$ que tiene igual número de comunidades vegetales pero que agrupa un menor de UGOs. Otra diferencia entre estas unidades es el número de especies exclusivas (Elementos Florísticos); en la Unidad I se registran 332 mientras que en la Unidad IV se registran más del doble (764), siendo ésta última la unidad florística con mayor número de especies exclusivas. En la Unidad III se registra la mayor riqueza de especies y comunidades vegetales, mientras que la Unidad II es la más pobre en riqueza florística, pues sus ocho UGOs registran el menor número de especies exclusivas y de comunidades vegetales (Cuadro 4; Figura 5).

\section{Discusión}

La contribución de taxa por familia a lo largo de la FVT es heterogénea. Las familias mejor representadas son Asteraceae, Poaceae y Fabaceae, probablemente como conse- cuencia de ser las familias que en la FVT tienen uno de sus principales centros de diversificación (Rzedowski, 1978), además de ser las más diversas y de las que se tienen mayor número de registros en México. La familia Asteraceae es la más diversa de las regiones templadas de México (Rzedowski, 1978; Villaseñor y Ortiz, 2007).

La riqueza de especies encontrada en las UGOs también señala que la diversidad local (diversidad alfa) en cada UGO en que fue dividida la FVT no es uniforme. Lo anterior permite entender la alta diversidad beta que se observa en esta provincia, responsable de la alta riqueza florística de la zona de estudio. Sin embargo, es necesario documentar mejor la información de su flora, pues algunas partes de su territorio muestran vacíos de información o una deficiencia de ella que debería llenarse con más trabajo de campo.

La mayoría de las especies (92\%) se distribuyen en menos de 15 UGOs. Este patrón puede deberse al relieve tan diverso observado en la FVT; la provincia incluye valles, cuencas, planicies y desde luego los geomorfos más abundantes que son las montañas (Ferrusquía-Villafranca, 2007). Esta diversidad morfotectónica, junto con el clima que es también muy diverso, generan barreras a distancias cortas en esta región, repercutiendo en la distribución de las especies. Otra explicación del alto número de especies restringidas en la FVT podría ser la distribución de muchas especies que se reparten con otras zonas montañosas vecinas. Con tales cadenas montañosas la FVT forma zonas complejas, las cuales apoyan el papel que históricamente ha jugado como corredor biológico; conectando, por ejemplo, la Sierra Madre Occidental con la Sierra Madre Oriental y viceversa (Villaseñor y Ortiz, 2007).

Los resultados apoyan afirmaciones previas de que la flora de la FVT es característica de los bosques templados, como ha sugerido Rzedowski (1978). Este componente, junto con el asociado a los bosques tropicales caducifolios y subcaducifolios, conforman los tipos de vegetación que ocupan la mayor área con cobertura vegetal en la FVT, superando considerablemente a los demás (Cuadro 3). Desafortunadamente, el área que ocupaban originalmente estos tipos de vegetación se ha visto reducida de manera considerable, repercutiendo en el área de distribución de las especies. Los datos indican que en los polígonos con algún tipo de vegetación solamente se registraron 27,116 sitios de recolecta, mientras que 61,823 se registraron en las zonas urbanas, agrícolas, ganaderas, etc. Lo anterior es muestra de cómo se ha reducido el área de distribución de las especies en la FVT, al quedar compactada la diversidad en pequeños fragmentos con vegetación remanente.

El pastizal inducido es uno de los tipos de vegetación (INEGI, 2005) que actualmente tiene la mayor cobertura y donde se registra una alta riqueza de especies (Cuadro 3). Estos datos sugieren que los tipos de vegetación primaria han sido fuertemente transformados por las actividades humanas y actualmente se encuentran como vegetación secun- 


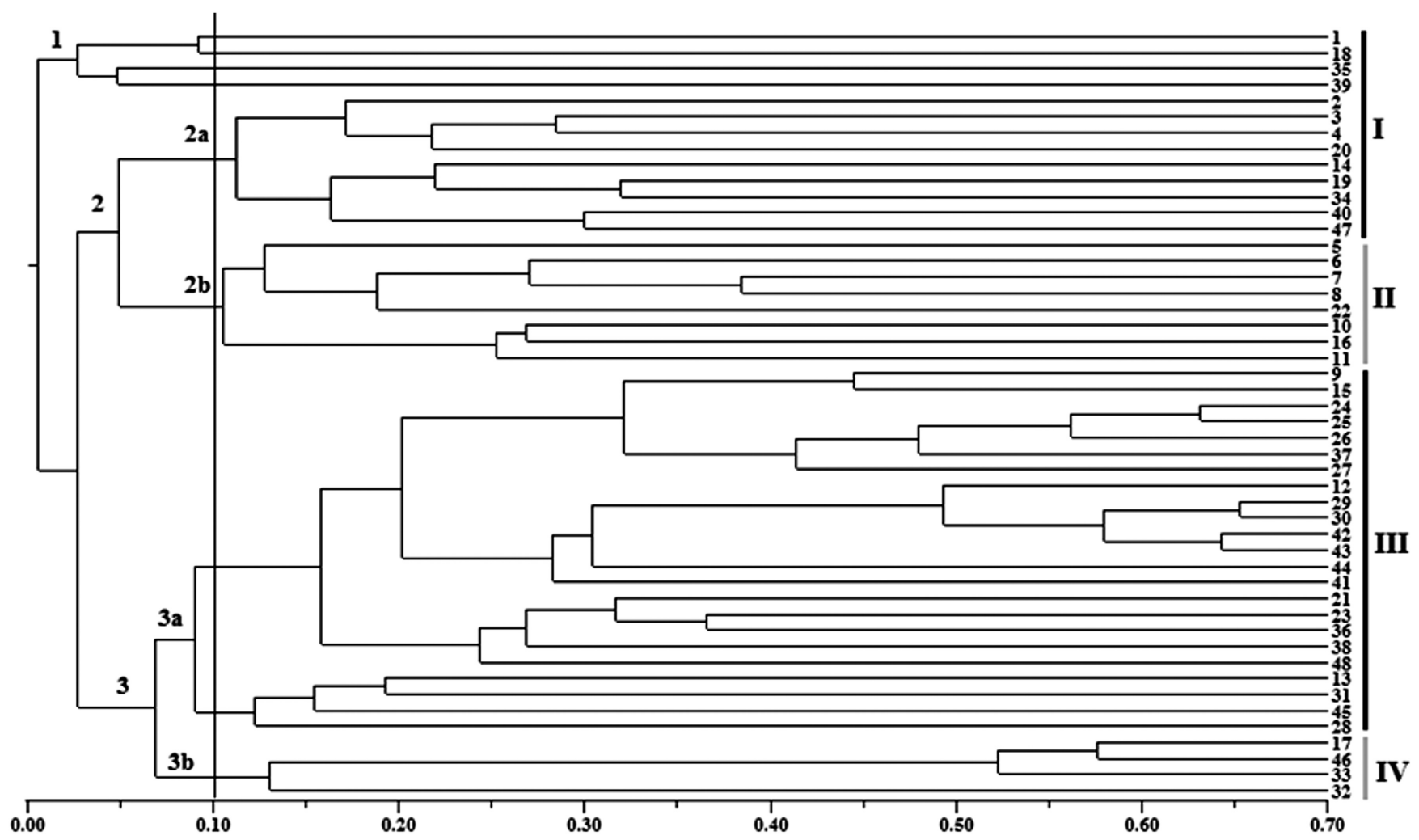

Figura 4. Valores de similitud encontrados entre las UGOs en que se dividió la FVT. Coeficiente de similitud: Sorensen-Dice; método de agrupamiento: Enlace Completo. Los números romanos indican las unidades florísticas identificadas (ver Cuadro 4).

daria o transformada. Algunos de estos sitios probablemente están funcionando como reservorios o refugios de la flora característica de los tipos de vegetación primaria circundantes. La distribución de especies en tipos de vegetación primaria indica que los bosques templados (pino-encino, oyamel, húmedos de montaña) son los tipos de vegetación en donde se concentran la mayoría de las especies que se distribuyen en la FVT (Cuadro 3).

De acuerdo con Birks (1976), los elementos florísticos, definidos como el conjunto de especies vegetales que se distribuyen en un área determinada (unidades florísticas), pueden evaluarse de dos maneras: (1) como el conjunto total de taxa de una unidad florística con distribución en otras unidades (especies compartidas) y (2) como el conjunto de taxa exclusivos de una determinada unidad florística. En este análisis consideramos la segunda opción para definir los elementos florísticos, por lo que los cuatro elementos definidos corresponden con las unidades florísticas determinadas (cuadro 4). El Elemento Florístico I incluye 332 especies distribuidas donde la FVT hace contacto con la Costa del Pacífico, relacionado con 22 dominios climáticos y 14 tipos de vegetación. Por la extensión del área de distribución (Unidad florística I) de este elemento, podemos inferir que son especies compartidas con otras provincias como la Depresión del Balsas y la Costa del Pacífico, las cuales están conformadas por elementos de la región Neotropical. En cambio, las 31 especies del Elemento Florístico II, están relacionadas con un mayor número de dominios climáticos (33) y un menor número de tipos de vegetación. La distribución de este elemento en la parte norte, donde la FVT entra en contacto con la provincia del Altiplano, es una de las zonas más secas y donde prosperan especies típicas de matorrales xerófilos, con afinidades a la región Neártica. El Elemento Florístico III es el que tiene mayor cobertura dentro de la FVT, no sólo por la extensión de su área (Unidad florística III), sino también por estar relacionado con 25 dominios climáticos y 16 diferentes tipos de vegetación. Las especies que lo constituyen tienen afinidades con ambientes templados localizados en la parte central de la FVT y donde se encuentra la cordillera montañosa con las mayores altitudes de México.

Las unidades florísticas definidas no sólo difieren por la cantidad de UGOs que las forman sino también por el total de especies que contienen (elementos florísticos sensu Birks, 1976). El agrupamiento de las UGOs con base en los valores de similitud revela una división de la FVT en dos zonas definidas longitudinalmente, una oriental y otra occidental (Figura 5). Los resultados también indican que las especies se pueden dividir en tres grupos: (1) aquellas que prosperan preferentemente en las condiciones de aridez que se presen- 


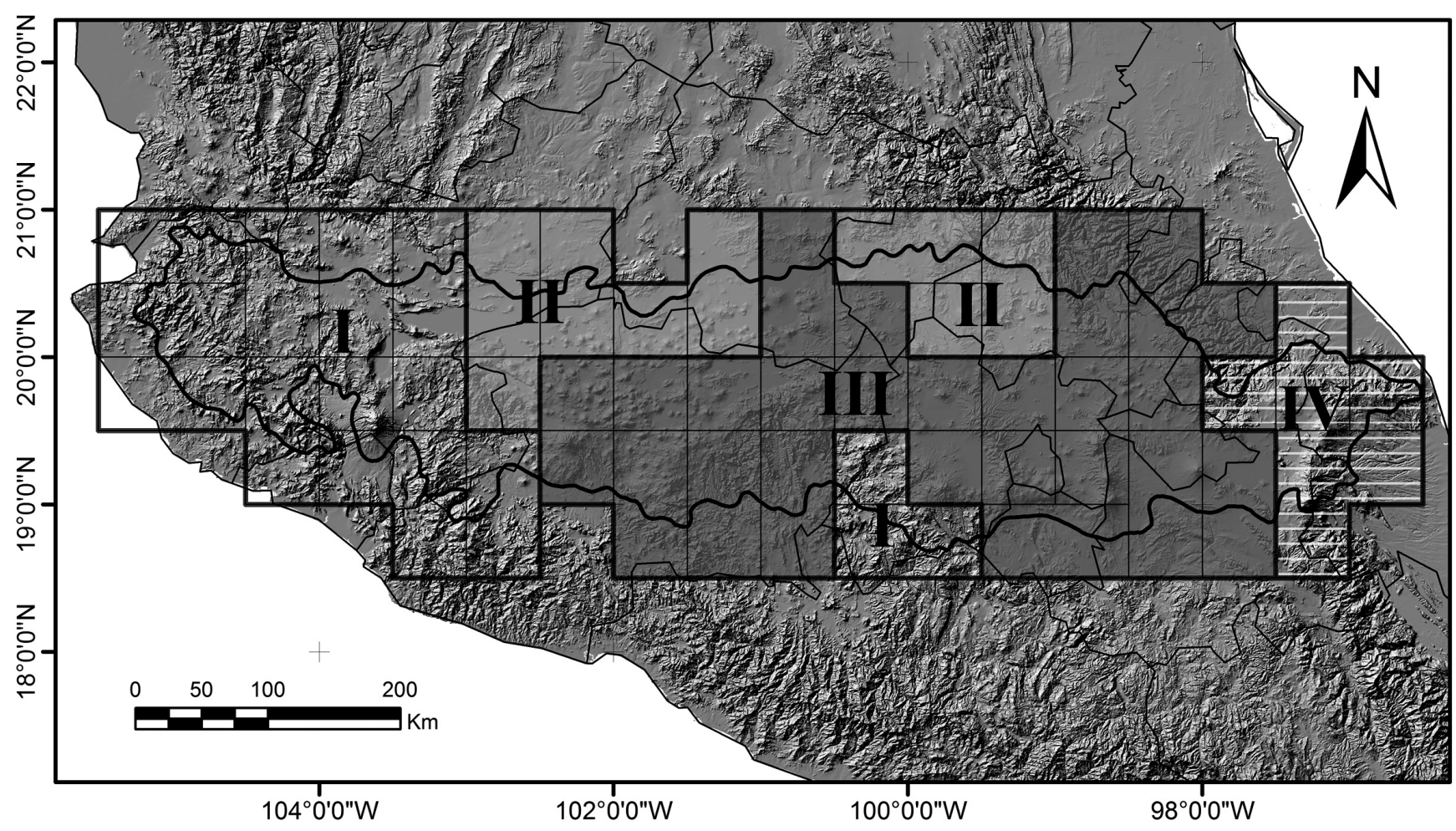

Figura 5. Unidades florísticas definidas con la similitud encontrada entre las UGOs en que se dividió la FVT.

tan en la parte norte, en donde la FVT entra en contacto con la Altiplanicie Mexicana (Unidad florística II), (2) aquellas que se distribuyen básicamente en hábitats con climas templados, localizados en la parte central de la FVT (Unidad florística III) y (3) aquellas que se distribuyen en climas más cálidos, pero con un contraste importante de humedad ambiental, la porción occidental (Unidad I) conspicuamente más seca que la porción oriental (Unidad IV).

Legendre y Legendre (1998) recomiendan que las unidades florísticas tengan una continuidad espacial y, por lo tanto, se deben unir aquellas que no difieran ostensiblemente en su composición florística pero que se encuentren separadas geográficamente. En este estudio la Unidad I forma dos grupos con importantes niveles de similitud florística (Figura 4) pero que se encuentran separados geográficamente (Figura 5). La división de esta Unidad I coincide en general con la distribución de la cubierta vegetal y con los rasgos climáticos presentes en las provincias florísticas propuestas por Rzedowski (1978). Las especies de la Unidad I prosperan en las condiciones ambientales características de las provincias Costa del Pacifico y Depresión del Balsas. Estas dos provincias, al igual que la Unidad I están divididas por las Serranías Meridionales (Unidad florística III), provincia formada por especies que se distribuyen en hábitats con climas templados.

Alcántara y Paniagua (2007) reportan 63 especies endémicas de la FVT. Algunas de ellas (16) tienen su distribución de manera exclusiva en una de las cuatro unidades definidas en este estudio. En la Unidad I se distribuyen tres de estas 16 especies (Epidendrum examinis S.Rosillo, Graptopetalum fruticosum Moran y Verbesina culminicola McVaugh); en la Unidad III diez (Achaetogeron mexicanus (A.Gray) DeJong, Cirsium pazcuarense (Kunth) Spreng, Echeandia gracilis Cruden, Festuca rzedowskiana E.B.Alexeev, Lychnis mexica$n a$ Rose, Muhlenbergia breviseta Griseb. ex E.Fourn, Plantago tolucensis Pilg., Poa orizabensis Hitchc., Salix mexicana Seemen, Sedum mínimum Rose) y en la Unidad IV tres más (Calamagrostis eriantha (Kunth) Steud., Echeveria subalpina Rose et Purpus, Oreomyrrhis orizabae I.M.Johnst.). La Unidad II es la única en la que no se registran especies endémicas exclusivas, pues las demás especies endémicas se comparten entre dos o más de las unidades florísticas.

Datos reportados para especies de la tribu Senecioneae (Asteraceae) y para briófitas (Villaseñor et al., 2006) señalan que el endemismo en la FVT es mayor a lo que reportan Alcántara y Paniagua (2007). Villaseñor et al. (2006) describen cuatro zonas de riqueza de especies (hotspots) en la FVT, las cuales coinciden con las unidades florísticas definidas en este análisis; una de ellas está incluida en la Unidad I, otras dos en la Unidad III y la última en la Unidad IV. Estas coincidencias, sugieren que el endemismo es un elemento importante para ser considerado en propuestas de regionalización de áreas.

Las unidades florísticas definidas en este trabajo pueden 
ser contrastadas con las propuestas planteadas con otros grupos taxonómicos. Por ejemplo, Sánchez-Cordero et al. (2005) proponen tres zonas de concentración de especies de mamíferos en la FVT, una oriental, otra central y otra occidental; por otra parte, Escalante et al. (2007), con base en datos de distribución potencial de mamíferos, reconocen dos áreas, una oriental y otra occidental. Ambos trabajos tienen ciertas coincidencias con la distribución de las unidades florísticas delimitadas en este análisis (Figura 5), en la que latitudinalmente se distinguen también tres zonas. Sin embargo, longitudinalmente se aprecian diferencias, pues ningún otro estudio reconoce la parte norte y sur de la FVT (aquí denominadas Unidades II y III). Las diez regiones avifaunísticas identificadas en la FVT por Navarro-Sigüenza et al. (2007) coinciden en parte con las reconocidas en este trabajo, aunque los autores utilizan polígonos diferentes (provincias biogeográficas) a los aquí utilizados (provincias geomorfológicas). Moore (1945), reconoce cinco distritos avifaunísticos en la FVT; que coinciden geográficamente con los reconocidos por Torres y Luna (2007) que, con base en otros grupos taxonómicos, también delimitan cinco distritos en la FVT. Aunque estos cinco distritos son delimitados utilizando áreas mayores a las del polígono empleado en este trabajo, son coincidentes con las unidades florísticas aquí delimitadas.

Los esfuerzos realizados para reconocer áreas de concentración de especies que permitan regionalizar la FVT, indican que hay demasiada complejidad para lograr un sólo objetivo, debido a la heterogeneidad mostrada por la distribución de la flora y la fauna en esta zona del país, considerada como una de las más importantes por su riqueza biológica. Sin embargo, el reconocimiento de áreas biogeográficas con todos estos trabajos permitirá planificar mejores estrategias para delimitar áreas prioritarias de conservación.

\section{Agradecimientos}

Este trabajo se realizó como parte de un proyecto de investigación del primer autor dentro de sus estudios de doctorado, por lo que agradece al Posgrado en Ciencias Biológicas de la UNAM y a CONACYT (Beca 186550). Un agradecimiento también a Mayra M. Hernández M. y Humberto Macías C. por su asesoría técnica en el manejo de los datos y del sistema de información geográfica. Agradecemos además a los revisores (anónimos) que corrigieron y enriquecieron el contexto del manuscrito.

\section{Literatura citada}

Akeroyd J. y Synge H. 1992. Higher plant diversity. En: Groombridge B. Ed. Global Biodiversity: Status of the Earth's Living Resources, pp. 64-87, Chapman \& Hall, Londres.

Alcántara O. y Paniagua M. 2007. Patrones de distribución y conservación de plantas endémicas. En: Luna I., Morrone J.J. y Es- pinoza D. Eds. Biodiversidad de la Faja Volcánica Transmexicana, pp. 421-438, Comisión Nacional para el Conocimiento y Uso de la Biodiversidad, Universidad, Nacional Autónoma de México, México, D.F.

Alcorta G.R. 1964. Caminos de México-esquema geográfico de México. En: Atlas Gooddrich Euzkadi. México, D.F.

Arriaga L., Aguilar C., Espinosa D. y Jiménez R. 1997. Regionalización Ecológica y Biogeográfica de México. Comisión Nacional para el Conocimiento y Uso de la Biodiversidad, México, D.F.

Birks H.J.B. 1976. The distribution of European Pteridophytes: a numerical analysis. New Phytologist 77:257-287.

Casas-Andreu G. y Reyna-Trujillo T. 1990. Herpetofauna (Anfibios y Reptiles). En: Instituto de Geografía Ed. Atlas Nacional de México Tomo II, IV.8.6, Universidad Nacional Autónoma de México, México, D.F.

Clausen R.T. 1959. Sedum of the Trans-Mexican Volcanic Belt: An Exposition of Taxonomic Methods. Cornell University Press, Nueva York.

CONABIO (Comisión Nacional para el Conocimiento y Uso de la Biodiversidad). 1997. "Provincias Geomorfológicas de México". Escala 1:4,000,000. México, D.F.

Contreras-Medina R. y Eliosa-León H. 2001. Una visión panbiogeográfica preliminar de México. En: Llorente J. y Morrone J.J. Eds. Introducción a la Biogeografía en Latinoamérica: Conceptos, Teorías, Métodos y Aplicaciones, pp. 197-211, Instituto de Ecología, ECOSUR. Universidad Nacional Autónoma de México, México, D.F.

Cuanalo de la Cerda H., Ojeda-Trejo E., Santos-Ocampo A. y. Ortíz-Solorio C.A. 1989. Provincias, Regiones y Subregiones Terrestres. Colegio de Postgraduados, Centro de Edafología, Texcoco.

Daniel W.W. 1982. Bioestadística. Base para el Análisis de las Ciencias de la Salud. Limusa, México, D.F.

Darlington P.J. 1957. Zoogeography: The Geographical Distribution of Animals. John Wiley and Sons, Nueva York.

Delgadillo C., Villaseñor J.L. y Dávila P. 2003. Endemism in the Mexican flora: a comparative study in three plant groups. Annals of the Missouri Botanical Garden 90:25-34.

Demant A. 1978. Características del Eje Neovolcánico Transmexicano y sus problemas de interpretación. Revista Instituto de Geología 2:172-187.

Escalante T., Morrone J.J. y Rodríguez G. 2005. Las provincias biogeográficas del componente Mexicano de Montaña desde la perspectiva de los mamíferos continentales. Revista Mexicana de Biodiversidad 76:199-205.

Escalante T., Rodríguez G., Gámez N., León-Paniagua L., Barrera O. y Sánchez-Cordero V. 2007. Biogeografía y conservación de los mamíferos. En: Luna I., Morrone J.J. y Espinoza D. Eds. Biodiversidad de la Faja Volcánica Transmexicana, pp. 485-502, Comisión Nacional para el Conocimiento y Uso de la Biodiversidad, Universidad Nacional Autónoma de México, México, D.F.

ESRI (Environmental Scientific Research Institute) 2000. ArcView 3.2. ESRI, Redlans.

Fa J.E. y Morales L.M. 1991. Mammals and protected areas in the Trans-Mexican Neovolcanic Belt. En: Mares M.A. y Schmidly D.J. Eds. Latin American Mammalogy: History, Biodiversity, and Conservation, pp. 199-226, University of Oklahoma Press, Norman, 
Farjon A. y Styles B.T. 1997. Pinus (Pinaceae). Flora Neotropica Monograph 75. The New York Botanical Garden, Nueva York.

Ferrari L. 2000. Avances en el conocimiento de la Faja Volcánica Transmexicana durante la última década. Boletín de la Sociedad Geológica de México 53:84-92.

Ferrari L., López-Martínez M., Aguirre-Díaz G. y Carrasco-Nuñez G. 1999. Space-time patterns of Cenozoic arc volcanism in central Mexico: From the Sierra Madre Occidental to the Mexican Volcanic Belt. Geology 27:303-306.

Ferrusquía-Villafranca I. 1990. Regionalización biogeográfica. En: Instituto de Geografía Ed. Atlas Nacional de México Tomo II, IV.8.10, Universidad Nacional Autónoma de México, México, D.F.

Ferrusquía-Villafranca I. 2007. Ensayo sobre la caracterización y la significación biológica. En: Luna I., Morrone J.J. y Espinoza D. Eds. Biodiversidad de la Faja Volcánica Transmexicana, pp. 7-23, Comisión Nacional para el Conocimiento y Uso de la Biodiversidad, Universidad Nacional Autónoma de México, México, D.F.

Goldman E.A. y Moore R.T. 1945. The biotic provinces of Mexico. Journal of Mammalogy 26:347-360.

Gómez-Tuena A., Orozco-Esquivel M.T. y Ferrari L. 2005. Petrogénesis ígnea de la Faja Volcánica Transmexicana. Boletín de la Sociedad Geológica de México 57:227-285.

Greenland D. 2005. Mountain and valley winds. En: Oliver J.E. Ed. Encyclopedia of World Climatology, pp. 516-523, Springer, Dordrecht.

Halffter G. 1976. Distribución de los insectos en la zona de transición mexicana: relaciones con la entomofauna de Norteamérica. Folia Entomologica Mexicana 35:1-64.

Halffter G. 2003. Biogeografía de la entomofauna de montaña de México y América Central. En: Morrone J.J. y Llorente-Bousquets J. Eds. Una Perspectiva Latinoamericana de la Biogeografía, pp. 87-97, Las Prensas de Ciencias, Universidad Nacional Autónoma de México, México, D.F.

INEGI. 2005. Conjunto de Datos Vectoriales de Uso de Suelo y Vegetación. Escala 1:250,000, Serie III (Conjunto Nacional). Dirección General de Geografía, Instituto Nacional de Estadística, Geografía e Informática.

Legendre P. y Legendre L. 1998. Numerical Ecology. $2^{\text {nd }}$ ed., Elsevier Publishers, Amsterdam.

López-Ramos E. 1979. Geología de México Tomo III. Edición privada, México, D.F.

Lugo-Hubp J. 1990. El relieve de la República Mexicana. Revista del Instituto de Geología 9:82-111.

McLaughlin S.P. 1994. Floristic plant geography: the classification of floristic areas and floristic elements. Progress in Physical Geography 18:185-208.

Mittermeier R.A. 1988. Primate diversity and the tropical forest. En: Wilson E.O. y Peter F.M. Eds. Biodiversity, pp. 145- 154, National Academic Press, Washington, DC.

Moore R.T. 1945. The transverse volcanic biotic province of central México and its relationship to adjacent provinces. Transactions of the San Diego Society of Natural History 10:217-236.

Mooser F. 1972. The Mexican Volcanic Belt - Structure and tectonics. Geofisica Internacional 12:55-70.

Morafka D.J., Adest G.A., Reyes L.M., Aguirre G. y Lieberman S.1992. Differentiation of North American deserts: A phylogenetic evaluation of a vicariante model. En: Darwin S.P. y Welden A.L. Ed. Biogeography of Mesoamerica, pp. 195-226,
Tulane University, Nuevo Orleáns.

Morán-Zenteno D. 1984. Geología de la República Mexicana. Secretaría de Programación y Presupuesto, Instituto Nacional de Estadística, Geografía e Informática, México, D.F.

Morrone J.J. 2001. Toward a cladistic model for the Caribbean subregion: Delimitation of areas of endemism. Caldasia 23:43-76.

Morrone J.J. 2005. Hacia una síntesis biogeográfica de México. Revista Mexicana de Biodiversidad 76:207-252.

Morrone J.J. 2006. Biogeographic areas and transition zones of Latin America and the Caribbean Islands based on panbiogeographic and cladistic analyses of the Entomofauna. Annual Review of Entomology 51:467-494.

Morrone J.J. y Márquez J. 2003. Aproximación a un atlas biogeográfico mexicano: componentes bióticos principales y provincias biogeográficas. En: Morrone J. J. y Llorente-Bousquets J. Eds. Una Perspectiva Latinoamericana de la Biogeografía, pp. 217-220, Las Prensas de Ciencias, Universidad Nacional Autónoma de México, México, D.F.

Navarro-Sigüenza A.G., Lira-Noriega A., Peterson A.T., Oliveras de Ita A. y Gordillo-Martínez A. 2007. Diversidad, endemismo y conservación de las aves. En: Luna, I., Morrone J.J. y Espinoza D. Eds. Biodiversidad de la Faja Volcánica Transmexicana, pp. 461-483, Comisión Nacional para el Conocimiento y Uso de la Biodiversidad, Universidad Nacional Autónoma de México, México, D.F.

Ochoa-Tejeda V. 2001. Geomorfología, clima y vegetación del Valle de Tehuacán-Cuicatlán Pue.-Oax. México. Tesis de licenciatura. Facultad de Ciencias. Universidad Nacional Autónoma de México. México, D. F. 80 pp.

Ortega-Gutiérrez F., Mitre-Salazar L.M., Roldán-Quintana J., Aranda-Gómez J.J., Morán-Zenteno D., Alaniz-Álvarez S.A. y Nieto-Samaniego A. 1992. Carta Geológica de la República Mexicana, escala 1: 2,000,000, 5a. ed., Consejo de Recursos Naturales, Instituto de Geología, Universidad Nacional Autónoma de México, México, D.F.

Raisz E. 1964. Landforms of Mexico. 2nd ed. Geography Branch. Office of Naval Research, Cambridge.

Ramírez-Pulido J. y Castro-Campillo A. 1990. Regionalización mastofaunística (Mamíferos). En: Instituto de Geografía Ed. Atlas Nacional de México Tomo II, IV.8.8, Universidad Nacional Autónoma de México, México, D.F.

Rohlf F.J. 2002. NTSYS-PC 2.2. Numerical Taxonomy and Multivariate Analysis System. Exeter Software: Applied Biostatistics, Nueva York.

Rzedowski J. 1973. Geographical relationships of the flora of Mexican dry regions. En: Graham A. Ed. Vegetation and Vegetational History of Northern Latin America, pp. 61-72, Elsevier Scientific Company, Amsterdam.

Rzedowski J. 1978. Vegetación de México. Limusa, México, D.F.

Rzedowski J. 1991. Diversidad y orígenes de la flora fanerogámica de México. Acta Botanica Mexicana 14:3-21.

Rzedowski J. y Calderón de Rzedowski G. 1993. Datos sobre la dinámica de la flora fanerogámica del valle de México, con énfasis en especies narrativas raras, en peligro de extinción y aparentemente extintas. Acta Botanica Mexicana 25:81-108.

Rzedowski J. y Reyna-Trujillo T. 1990. Divisiones florísticas. En: Instituto de Geografía Ed. Atlas Nacional de México Tomo II. IV.8.3, Universidad Nacional Autónoma de México, México, D.F.

Sánchez-Cordero V., Cirelli V., Munguial M. y Sarkar S. 2005. 
Place prioritization for biodiversity content using species ecological niche modeling. Biodiversity Informatics 2:11-23.

Smith H.M. 1941. Las provincias bióticas de México, según la distribución geográfica de las lagartijas del género Sceloporus. Anales de la Escuela Nacional de Ciencias Biológicas 2:103-110.

Suárez-Mota M.E. 2006. Afinidades geográficas del valle de Tehuacán-Cuicatlán analizando las Asteraceae Mexicanas. Tesis de maestría. Facultad de Estudios Superiores Iztacala. Universidad Nacional Autónoma de México. México. 47 pp.

Suárez-Mota M.E. 2012. Regionalización ambiental y florística y conservación de la Provincia Biogeográfica del Eje Volcánico Transversal de México. Tesis de doctorado. Facultad de Estudios Superiores Iztacala. Universidad Nacional Autónoma de México. México. 129 pp.

Suárez-Mota M.E. y Villaseñor J.L. 2011. Las compuestas endémicas de Oaxaca, México: diversidad y distribución. Boletín de la Sociedad Botánica de México 88:55-66.

Torres M.A. y Luna I. 2007. Hacia una síntesis panbiogeográfica. En: Luna I., Morrone J.J. y Espinoza D. Eds. Biodiversidad de la Faja Volcánica Transmexicana, pp. 503-514, Comisión Nacional para el Conocimiento y Uso de la Biodiversidad, Universidad Nacional Autónoma de México, México, D.F.

UICN. 2001. Categorías y criterios de la lista Roja de la UICN. Versión 3.1. Comisión de Supervivencia de Especies de la UICN (Unión Internacional para la Conservación de la Naturaleza), Cambridge.

Recibido: 30 de mayo de 2012

Aceptado: 16 de agosto de 2012
Valencia-A. S. 2004. Diversidad del género Quercus (Fagaceae) en México. Boletín de la Sociedad Botánica de México 75:33-53.

Villaseñor J.L. 2003. Diversidad y distribución de las Magnoliophyta de México. Interciencia 28:160-167.

Villaseñor J.L. 2004. Los géneros de las plantas vasculares de la flora de México. Boletín de la Sociedad Botánica de México 75:105-135.

Villaseñor J.L. 2010. El Bosque Húmedo de Montaña en México y sus Plantas Vasculares: Catálogo Florístico-Taxonómico. Comisión Nacional para el Conocimiento y Uso de la Biodiversidad, Universidad Nacional Autónoma de México, México, D.F.

Villaseñor J.L. y Ortiz E. 2007. La familia Asteraceae. En: Luna I., Morrone J.J. y Espinoza D. Eds. Biodiversidad de la Faja Volcánica Transmexicana, pp. 289-310, Comisión Nacional para el Conocimiento y Uso de la Biodiversidad, Universidad Nacional Autónoma de México, México, D.F.

Villaseñor J.L., Delgadillo C. y Ortiz E. 2006. Biodiversity hotspots from a multigroup perspective: Mosses and Senecios in the Transmexican Volcanic Belt. Biodiversity and Conservation 15:4045-4058.

Villaseñor J.L., Maeda P., Rosell J.A y Ortiz E. 2007. Plant families as predictors of plant biodiversity in Mexico. Diversity and Distributions 13:871-876.

Vivó J.A. 1943. Los límites biogeográficos en América y la zona cultural mesoamericana. Revista Geográfica 3:109-131. 